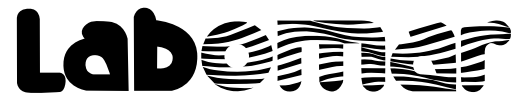

Arquivos de Ciências do Mar

\section{REGISTROS DE Gonioplectrus hispanus (CUVIER, 1828) (ACTINOPTERYGII: EPINEPHELIDAE) NO ESTADO DA BAHIA (NORDESTE DO BRASIL), OCEANO ATLÂNTICO OCIDENTAL}

\author{
Records of Gonioplectrus hispanus (cuvier, 1828) \\ (actinopterygii: epinephelidae) in Bahia state (northeastern \\ of Brazil), western Atlantic Ocean \\ Cláudio L. S. Sampaio', Paulo Roberto D. Lopes², \\ Jailza T. de Oliveira-Silva ${ }^{3}$, Priscilla Malafaia ${ }^{4}$, George Olavo ${ }^{5}$ \\ ${ }^{1}$ Professor adjunto. Universidade Federal de Alagoas. Unidade de Ensino de Penedo. Av. Beira Rio, s/n ${ }^{\circ}$, Centro \\ Histórico, Penedo - Alagoas. 57.000-000. E-mail: buiabahia@gmail.com \\ ${ }^{2}$ Professor assistente. Universidade Estadual de Feira de Santana, Departamento de Ciências Biológicas (Museu de \\ Zoologia, Divisão de Peixes), Avenida Transnordestina, s/no. (km 03 - BR-116), Feira de Santana - Bahia, 44.036-900. \\ E-mail: andarilho40@gmail.com \\ ${ }^{3}$ Bióloga. Univ. Est. de Feira de Santana, Dep. de Ciências Biológicas (Museu de Zoologia - Divisão de Peixes). \\ E-mail: jtosilva@yahoo.com.br \\ ${ }^{4}$ Mestre em Modelagem em Ciências da Terra e do Ambiente (Univ. Est. de Feira de Santana). \\ E-mail: priscillamalafaia@gmail.com \\ ${ }^{5}$ Professor assistente. Univ. Est. de Feira de Santana, Dep. de Ciências Biológicas, Lab. de Biologia Pesqueira. Avenida \\ Transnordestina, s/no. (km 03 - BR-116), Feira de Santana - Bahia, 44036-900. E-mail: georgeolavo@uol.com.br
}

\section{RESUMO}

A presença de Gonioplectrus hispanus (Cuvier, 1828) (Actinopterygii, Epinephelidae) é registrada para o estado da Bahia, costa nordeste do Brasil (Oceano Atlântico ocidental) com base em 8 exemplares medindo entre 247 e $292 \mathrm{~mm}$ de comprimento total (entre 207 e $241 \mathrm{~mm}$ de comprimento padrão). Dados sobre alimentação, reprodução, caracteres merísticos e morfométricos (com proporções corporais) são apresentados e comparados com a literatura.

Palavras-chave: garoupa, biologia, peixe de recifes profundos.

\section{ABSTRACT}

The presence of Gonioplectrus hispanus (Cuvier, 1828) (Actinopterygii, Epinephelidae) is recorded to Bahia state, northeastern coast of Brazil (Western Atlantic Ocean) based in 8 specimens measuring between 247 and $2920 \mathrm{~mm}$ of total length (between 207 and $241 \mathrm{~mm}$ of standard length).

Recebido em: 1\%/5/2016

Aprovado em: 6/6/2017

Publicado online em: 20/1/2018 
Diet, reproduction, morphometric (with corporal proportions) and meristic data are presented and compared with the literature about this species.

Key words: spanish flag grouper, biology, deep reef fish.

\section{INTRODUÇÃO}

Embora raro, Gonioplectrus hispanus (Cuvier, 1828) (Figuras 1 e 2) é conhecido pelos pescadores artesanais baianos como "ariacó de fundo" devido ao seu padrão de colorido. Atinge $300 \mathrm{~mm}$ de comprimento e é a única espécie do gênero, pertencendo à família Epinephelidae. Possui uma ampla distribuição geográfica, ocorrendo desde a Carolina do Norte (EUA) (registro de pós-larva pelágica) até a Ilha de Trindade, sudeste do Brasil, em fundos consolidados, entre 60 e 365 m de profundidade (Robins et al., 1986; Cervigón, 1991; Cervigón et al., 1992; Heemstra \& Randall, 1993; Heemstra et al., 2002; Moura, Menezes apud Menezes et al., 2003; McEachran \& Fechhelm, 2005; Nelson, 2006).

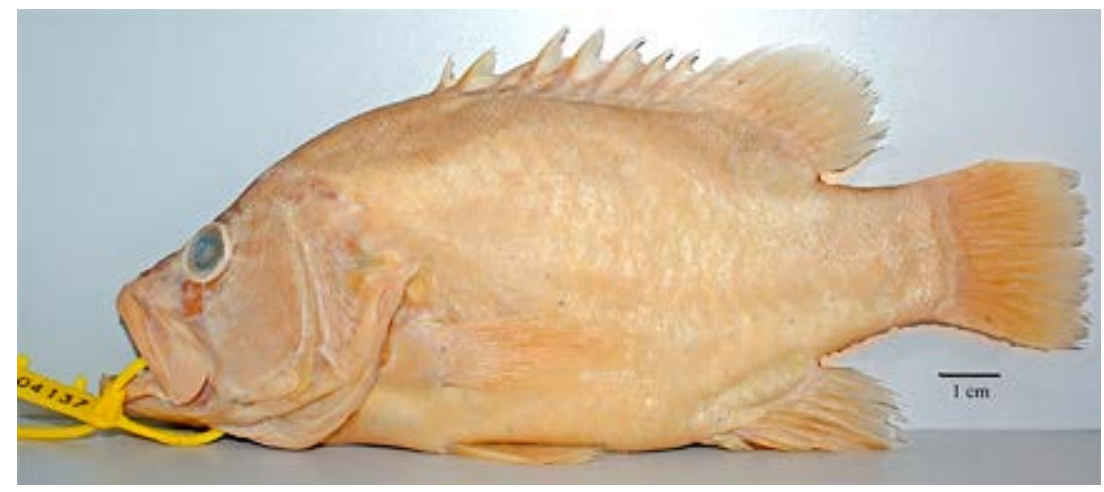

Figura 1 - Gonioplectrus hispanus, LIUEFS 4137 (285,0 mm de comprimento total).

Figura 2 - Gonioplectrus hispanus, ex. 02 (280,0 mm de comprimento total), poucas horas antes de sua captura, apresentando o colorido característico.

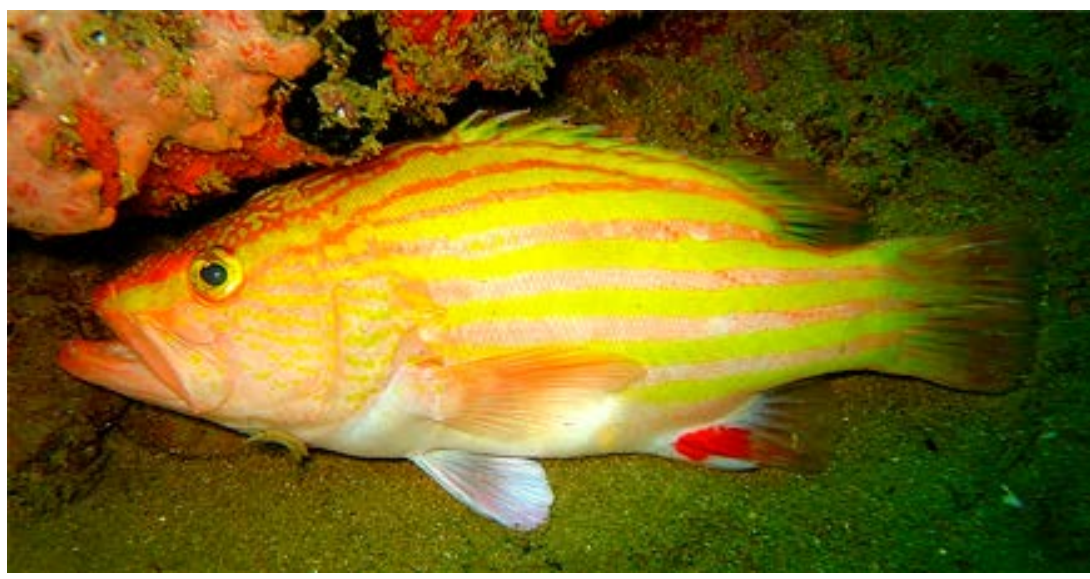

Devido ao pequeno porte e por habitar a região do talude continental, G. hispanus não é alvo de qualquer pescaria, sendo capturado acidentalmente (Graig et al., 2011). Contudo, devido ao vistoso colorido é bastante cobiçado pelos aquaristas, embora rara- 
mente seja registrada no comercio ornamental. Apesar dos esforços de alguns coletores profissionais de organismos ornamentais, apenas recentemente essa espécie tornou-se disponível no mercado internacional (Michael, 2004).

Este trabalho fornece informações sobre G. hispanus para o Brasil considerando o reduzido conhecimento disponível sobre esta espécie ao longo de sua área de distribuição (Graig et al., 2011) bem como amplia o número de exemplares depositados em coleções científicas brasileiras.

\section{MATERIAL E MÉTODOS}

O material examinado foi capturado durante pescarias artesanais e esportivas com anzol e linha ao longo do município de Salvador (estado da Bahia, Nordeste do Brasil). Dos oito exemplares aqui citados, cinco estão conservados em álcool 70\% e depositados na coleção da Divisão de Peixes do Museu de Zoologia, Departamento de Ciências Biológicas, da Universidade Estadual de Feira de Santana (MZUEFS, Bahia). Outros três exemplares foram apenas examinados e fotografados no momento do seu desembarque no porto da Barra (Salvador).

As medidas foram efetuadas no lado direito do corpo de cada exemplar com auxílio de paquímetro com precisão de $0,05 \mathrm{~mm}$ (exceto para comprimentos total e padrão quando utilizou-se ictiômetro e régua com precisão de 1,0 mm).

\section{RESULTADOS}

Foram examinados oito exemplares de G. hispanus oriundos do talude continental, região popularmente conhecida pelos pescadores como "paredes", entre 110 e $130 \mathrm{~m}$ de profundidade. Os indivíduos mediam entre 247 e $292 \mathrm{~mm}$ de comprimento total.

Além desses exemplares sabe-se, pelo menos, de mais dois que foram apenas fotografados durante seu desembarque, sendo um na região de Valença e outro em Arembepe, Camaçari, ambos no litoral baiano.

Quanto à alimentação, o exemplar LIUEFS 3313, encontrava-se com o estômago evertido; no LIUEFS 3418 o estômago estava rompido dentro da cavidade abdominal; no LIUEFS 4137 havia matéria orgânica digerida enquanto nos exemplar LIUEFS 4652 e nos demais exemplares examinados no momento do desembarque foram observados matéria orgânica digerida e material não identificado.

O exemplar LIUEFS 3418 era uma fêmea com gônadas em maturação enquanto o LIUEFS 4652 era uma fêmea com gônadas desenvolvidas (ocupando quase metade da cavidade abdominal), mas com ovócitos de pequeno diâmetro, provavelmente ainda em maturação. O exemplar LIUEFS 4137 era um macho com gônadas em maturação e no LIUEFS 3313 não foram visualizadas gônadas.

\section{DISCUSSÃO}

O primeiro registro de G. hispanus no Brasil ocorreu em 1998 e foi feito com base em um único exemplar que media $205 \mathrm{~mm}$ de comprimento padrão. O indivíduo foi captu-

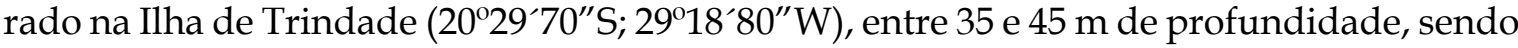
essa a menor profundidade de coleta conhecida (Teixeira \& Gasparini, 1999). 
No que concerne aos caracteres merísticos, Cervigón (1991), Cervigón et al. (1992), Hoese \& Moore (1998) e Heemstra et al. (2002) afirmam que G. hispanus possui 8 espinhos e 13 raios na nadadeira dorsal e 3 espinhos e 7 raios na nadadeira anal. Os mesmos valores foram obtidos para os exemplares aqui analisados. Porém, variações foram observadas para o número de escamas da linha lateral, de escamas em séries laterais no corpo, de escamas entre o sexto espinho da dorsal e a linha lateral, de rastros branquiais tanto no ramo inferior como no superior, de espinhos e raios da dorsal, raios das peitorais e nas relações de comprimento da cabeça/comprimento padrão e altura do corpo/largura do corpo quando comparadas com Cervigón (1991), Heemstra \& Randall (1993) e Heemstra et al. (2002). Tais diferenças contribuem para um melhor conhecimento sobre a variação de caracteres merísticos e morfométricos de G. hispanus ao longo de sua área de distribuição geográfica, especialmente para o Brasil, onde poucas informações e exemplares em coleções estão disponíveis.

Não existem informações disponíveis sobre a biologia de G. hispanus (Heemstra \& Randall, 1993; Graig et al., 2011). Na Ilha Branca (Venezuela), Cervigón (1991) examinou seis exemplares de G. hispanus medindo entre 171 e $222 \mathrm{~mm}$ de comprimento total e afirma que dados sobre esta espécie são muito escassos na literatura.

Heemstra et al. (2002) citam que um exemplar de G. hispanus com $170 \mathrm{~mm}$ de comprimento padrão foi identificado como macho maduro e que nenhuma outra informação sobre sua biologia está disponível.

Neste estudo, o único exemplar macho examinado neste estudo não estava sexualmente maduro e media $233 \mathrm{~mm}$ de comprimento padrão. Porém, três fêmeas (jamais citadas para G. hispanus) foram identificadas na presente pesquisa, todavia aparentemente nenhuma delas estava madura devido ao pequeno diâmetro dos ovócitos.

Quanto à dieta, os dados apresentados nesta pesquisa não são consistentes. Não foram encontradas informações na literatura, mas, como a maioria dos Epinephelidae, $G$. hispanus possui hábitos carnívoros, consumindo principalmente peixes e crustáceos (Heemstra et al., 2002). Os exemplares aqui analisados foram coletados durante o dia, com iscas artificais de profundidade, com formato de pequenos peixes (jumping jigs) e pedaços de sardinhas (Harengula sp.). Devido à mudança brusca da profundidade durante as capturas, os estômagos dos exemplares examinados encontravam-se evertidos, embora um pescador tenha relatado que um exemplar coletado em Arembepe (não examinado) havia regurgitado um pequeno camarão vermelho. Esses dados, associados às iscas utilizadas, sugerem uma dieta composta de pequenos peixes e crustáceos.

Esta espécie não é importante como alimento devido à sua raridade e reduzido porte (Heemstra \& Randall, 1993; Heemstra et al., 2002) e raramente é capturado acidentalmente (Graig et al., 2011). Devido ao seu atraente colorido é uma espécie valiosa para o aquarismo, embora seu habitat em águas profundas pode tornar difícil mantê-lo vivo após a captura (Heemstra et al., 2002). Trata-se de uma espécie rústica, necessitando de aquários com muitas tocas e águas relativamente frias $\left(12,5\right.$ a $\left.22^{\circ} \mathrm{C}\right)$, podendo compartilhar o espaço com outras espécies agressivas ou de maior porte e predar peixes menores e crustáceos (Michael, 2004). As únicas coletas conhecidas foram feitas em $91 \mathrm{~m}$ de profundidade, contudo sem precisar a localidade, sendo não apenas um mergulho perigoso, mas demorado e caro, fazendo seus preços serem elevados (Michael, 2004). Apesar dos elevados preços praticados no mercado ornamental internacional, não há qualquer registro de coleta para esses fins ou mesmo observações no ambiente natural dessa espécie no Brasil (Feitosa et al., 2010; Pereira-Filho et al., 2011; Pinheiro et al., 2015; Sampaio, obs. pess.). A ausência desses 
registros é devido às dificuldades em realizar tais imersões. Utilizando equipamentos de mergulho sofisticados, coletores profissionais observaram que G. hispanus é mais abundante em áreas com muitas tocas e abrigos, onde foram registrados nadando de ponta cabeça, com o ventre direcionado para o teto das tocas, buscando abrigo no fundo dessas e por entre invertebrados sésseis (Michael, 2004).

AGRADECIMENTOS: Aos pescadores do Rio Vermelho (Salvador, Bahia) e ao Sr. Gianpaolo Harfush (Dive Bahia) pela doação dos exemplares utilizados neste estudo, às universidades Federal de Alagoas (UFAL) e Estadual de Feira de Santana (UEFS), pelo auxílio proporcionado para a realização deste estudo.

Tabela 1 - Caracteres merísticos, morfométricos (expresso em $\mathrm{mm}$ ) e proporções corporais (\% do comprimento padrão, entre parênteses) de 8 exemplares de G. hispanus coletados na Bahia, Brasil ( $\mathrm{e}=$ nadadeira peitoral esquerda, $\mathrm{d}=\mathrm{nadadeira}$ peitoral direita, $\mathrm{D}=$ arco branquial direito, $\mathrm{E}=$ arco branquial esquerdo, ex. = exemplar).

\begin{tabular}{|c|c|c|c|c|c|c|c|c|}
\hline \begin{tabular}{|l|} 
Dados merísticos / \\
Dados morfométricos \\
\end{tabular} & $\begin{array}{c}\text { LIUEFSS } \\
3313 \\
\end{array}$ & LIUEFS 3418 & LIUEFS 4137 & LIUEFS 4652 & $\begin{array}{c}\text { LIUEFS } \\
14222 \\
\end{array}$ & ex. 01 & ex. 02 & ex. 03 \\
\hline Raios nad peitorais & $14(\mathrm{e})-17(\mathrm{~d})$ & $17(\mathrm{~d})-17(\mathrm{e})$ & $16(\mathrm{e})-17(\mathrm{~d})$ & $16(\mathrm{e})-16(\mathrm{~d})$ & $15(\mathrm{e})-15(\mathrm{~d})$ & $16(\mathrm{e})$ & $15(\mathrm{e})$ & $15(\mathrm{e})$ \\
\hline Espinho nad. pélvicas & $1(\mathrm{e}, \mathrm{d})$ & $1(\mathrm{e}, \mathrm{d})$ & $1(\mathrm{e}, \mathrm{d})$ & $1(\mathrm{e}, \mathrm{d})$ & $1(\mathrm{e}, \mathrm{d})$ & $1(\mathrm{e}, \mathrm{d})$ & $1(\mathrm{e}, \mathrm{d})$ & $1(\mathrm{e}, \mathrm{d})$ \\
\hline Raios nad. pélvicas & $5(\mathrm{e}, \mathrm{d})$ & $5(e, d)$ & $5(e, d)$ & $5(\mathrm{e}, \mathrm{d})$ & $5(e, d)$ & $5(e, d)$ & $5(\mathrm{e}, \mathrm{d})$ & $5(\mathrm{e}, \mathrm{d})$ \\
\hline Espinhos nad. dorsal & 8 & 8 & 8 & 8 & 9 & 9 & 9 & 9 \\
\hline Raios nad. dorsal & 13 & 13 & 13 & 12 & 12 & 12 & 11 & 12 \\
\hline Espinhos nad. anal & 3 & 3 & 3 & 3 & 3 & 3 & 3 & 3 \\
\hline Raios nad. anal & 7 & 7 & 7 & 7 & 7 & 7 & 7 & 8 \\
\hline Compr. total & 292,0 & 274,0 & 285,0 & 252,0 & 247,0 & 270,0 & 280,0 & 240,0 \\
\hline Compr. padrão & 241,0 & 222,0 & 233,0 & 210,0 & 207,0 & 220,0 & 235,0 & 210,0 \\
\hline Compr. cabeça & $111,5-46,3 \%$ & $113,6-51,2 \%$ & $112,5-48,3 \%$ & $95,2-45,3 \%$ & $\begin{array}{l}99,3- \\
48,0 \% \\
\end{array}$ & \begin{tabular}{|l|}
$100,0-$ \\
$45,4 \%$ \\
\end{tabular} & \begin{tabular}{|c|}
$110,0-$ \\
$46,8 \%$ \\
\end{tabular} & $\begin{array}{r}90,0- \\
42,8 \% \\
\end{array}$ \\
\hline Compr. focinho & $44,5-18,5 \%$ & $52,1-23,5 \%$ & $45,3-19,4 \%$ & $32,7-15,6 \%$ & $\begin{array}{l}45,0- \\
21,7 \% \\
\end{array}$ & \begin{tabular}{|l|}
$42,0-$ \\
$19,1 \%$ \\
\end{tabular} & \begin{tabular}{|l|}
$40,0-$ \\
$17,0 \%$ \\
\end{tabular} & $\begin{array}{l}39,0- \\
18,5 \% \\
\end{array}$ \\
\hline Compr. maxila superior & $46,4(19,2 \%)$ & $44,4(20,0 \%)$ & $47,6(20,4 \%)$ & $41,0(19,5 \%)$ & $\begin{array}{c}40,8 \\
(19,7 \%) \\
\end{array}$ & - & - & - \\
\hline Diâmetro orbital & $16,2(6,7 \%)$ & $15,3(6,9 \%)$ & $\begin{array}{c}17,7 \\
(7,6 \%) \\
\end{array}$ & $\begin{array}{c}13,3 \\
(6,3 \%) \\
\end{array}$ & $14,2(6,8 \%)$ & $\begin{array}{c}15,2 \\
(6,9 \%) \\
\end{array}$ & \begin{tabular}{|c|}
14,7 \\
$(6,2 \%)$ \\
\end{tabular} & $\begin{array}{c}12,8 \\
(6,1 \%) \\
\end{array}$ \\
\hline Compr. nad. peitoral (d) & $58,1(24,1 \%)$ & $60,3(27,2 \%)$ & $57,5(24,7 \%)$ & $53,9(25,7 \%)$ & $\begin{array}{c}53,3 \\
(25,7 \%) \\
\end{array}$ & - & - & - \\
\hline Compr. nad. pélvica (d) & $47,9(19,9 \%)$ & $44,5(20,0 \%)$ & $46,3(19,9 \%)$ & $35,8(17,0 \%)$ & $\begin{array}{c}39,5 \\
(19,1 \%) \\
\end{array}$ & - & - & - \\
\hline Altura corpo & $100,0(41,5 \%)$ & $86,2(38,8 \%)$ & $96,0(41,2 \%)$ & $83,0(39,5 \%)$ & $\begin{array}{c}77,3 \\
(37,3 \%) \\
\end{array}$ & - & - & - \\
\hline Largura interorbital & $21,8(9,0 \%)$ & $17,9(8,1 \%)$ & $21,6(9,3 \%)$ & $14,9(7,1 \%)$ & $14,0(6,8 \%)$ & - & - & - \\
\hline Compr. pré-dorsal & $103,1(42,8 \%)$ & $106,3(47,9 \%)$ & $\begin{array}{c}107,3 \\
(46,0 \%)\end{array}$ & $95,1(45,3 \%)$ & $\begin{array}{c}92,2 \\
(44,5 \%)\end{array}$ & - & - & - \\
\hline $\begin{array}{l}\text { Rastros branquiais: ramo } \\
\text { inferior }\end{array}$ & $15(\mathrm{D}, \mathrm{E})$ & $15(\mathrm{D}, \mathrm{E})$ & $16(\mathrm{D}, \mathrm{E})$ & $\begin{array}{c}15 \\
(\mathrm{D}, \mathrm{E})\end{array}$ & $\begin{array}{l}15(\mathrm{D}), \\
17(\mathrm{E})\end{array}$ & - & - & - \\
\hline $\begin{array}{l}\begin{array}{l}\text { Rastros branquiais: ramo } \\
\text { superior }\end{array} \\
\end{array}$ & $6(\mathrm{~d}, \mathrm{e})$ & $6(\mathrm{~d}, \mathrm{e})$ & $6(d), 5(e)$ & $6(\mathrm{~d}), 5(\mathrm{e})$ & $6(\mathrm{~d}, \mathrm{e})$ & - & - & - \\
\hline Largura corpo & 31,5 & 32,5 & 41,2 & 27,6 & 26,1 & - & - & - \\
\hline $\begin{array}{l}\text { No. (aprox.) escamas } \\
\text { linha lateral }\end{array}$ & $53-57$ & $48-50$ & $52-54$ & $53-55$ & $47-49$ & - & - & - \\
\hline $\begin{array}{l}\text { No. (aprox.) escamas } \\
\text { entre } 6^{\circ} \text {. espinho dorsal e } \\
\text { linha lateral }\end{array}$ & 5 & 6 & 5 & 6 & $5-6$ & - & - & - \\
\hline $\begin{array}{l}\text { No. (aprox.) séries } \\
\text { laterais escamas } \\
\end{array}$ & $65-82$ & $66-75$ & $68-80$ & $72-73$ & $78-79$ & - & - & - \\
\hline
\end{tabular}




\section{REFERÊNCIAS}

Cervigón, F. Los peces marinos de Venezuela. Volumen I. Fundación Científica Los Roques, 425 p., Caracas, 1991.

Cervigón, F.; Cipriani, R.; Fischer, W.; Garibaldi, L.; Hendrickx, M.; Lemus, A.J.; Márquez, R.; Poutiers, J.M.; Robaina, G. \& Rodriguez, B. Guia de campo de las espécies comerciales marinas y de águas salobres de la costa septentrional de Sur America. Organizacion de las Naciones Unidas para la Agricultura y la Alimentacion, Organizacion de las Naciones Unidas para la Agricultura y La Alimentacion, 595 p., Roma, 1992.

Graig, M.T.; Sadovy, Y.J. \& Heemstra, P.C. Groupers of the world: a field and market guide. CRC Press, Boca Ratón, Flórida, 2001.

Heemstra, P.C.; Anderson Jr., W.D. \& Lobel, P.S. Serranidae, pp. 1308-1369, in Carpenter, K.E. (ed.), The living marine resources of the Western Central Atlantic. Volume 2: bony fishes part 1 (Acipenseridae to Grammatidae). FAO Species Identification Guide for Fishery Purposes and American Society of Ichthyologists and Herpetologists Special Publication no. 5, p. 601-1374, Rome, 2002.

Heemstra, P.C. \& Randall, J.E. FAO species catalogue. Vol. 16. Groupers of the world (family Serranidae, subfamily Epinephelinae). An annotated and illustrated catalogue of the grouper, rockcod, hind, coral grouper and lyretail species known to date. FAO Fish. Synop., v. 16, n. 125, p. 1-382, 1993.

Hoese, H.D. \& Moore, R.H. Fishes of the Gulf of Mexico, Texas, Louisiana, and adjacent waters. $2^{\text {nd }}$ ed. Texas A\&M University Press, Texas, 1998.

McEachran, J.D. \& Fechhelm, J.D. Fishes of the Gulf of Mexico. Volume 2: Scorpaeniformes to Tetraodontiformes. University of Texas Press, Austin, 2005.

Moura, R.L. \& Menezes, N.A. Família Serranidae, pp. 75-77, in: Menezes, N.A.; Buckup, P.A., Figueiredo, J.L. (eds), Catálogo das especies de peixes marinhos do Brasil. Museu de Zoologia da Universidade de São Paulo, 159 p., São Paulo, 2003.

Nelson, J.S. Fishes of the world. $4^{\text {th }}$ ed. John Wiley \& Sons, 601 p., New Jersey, 2006.

Pereira-Filho, G.H.; Amado-Filho, G.M.; Guimarães, S.M.P.B; Moura, R.L.; Sumida, P.Y.G.; Abrantes, D.P.; Bahia, R.G.; Guth, A.Z.; Jorge, R.R. \& Francini Filho, R.B. Reef fish and benthic assemblages of the Trindade and Martim Vaz island group, southwestern Atlantic. Brazilian J. Oceanogr., v. 59, n. 3, p. 201-212, 2011.

Pinheiro, H.T.; Mazzei, E.; Moura, R.L.; Amado-Filho, G.M.; Carvalho-Filho, A.; Braga, A.C.; Costa, P.A.S.; Ferreira, B.P.; Ferreira, C.E.L.; Floeter, S.R.; Francini Filho, R.B.; Gasparini, J.L.; Macieira, R.M.; Martins, A.S.; Olavo, G.; Pimentel, C.R.; Rocha, L.A.; Sazima, I.; Simon, T.; Teixeira, J.B.; Xavier, L.B. \& Joyeux, J.-C. Fish biodiversity of the VitóriaTrindade Seamount Chain, Southwestern Atlantic: an updated database. PLoS ONE, v. 10, n. 3, p. e0118180, 2015.

Robins, C.R. Ray, G.C. \& Douglass, J. A field guide to Atlantic coast fishes of North America. Houghton Mifflin Company, 354p., Boston, 1986.

Teixeira, R.L. \& Gasparini, J.L. Gonioplectrus hispanus (Perciformes: Serranidae: Epinephelini). Nova ocorrência para a ictiofauna recifal brasileira, p. 65 in Resumos XIII Encontro Brasileiro de Ictiologia, Universidade Federal de São Carlos - Sociedade Brasileira de Ictiologia, 671 p., São Carlos, 1999. 